\title{
Prosthodontic Restoration of Vertical Dimension of Occlusion in Severly Worn Dentitions
}

\author{
Dr Abhishek Gupta*1 and Dr Parneet ${ }^{1}$ \\ Reader, Conservative dentistry and endodontic Maharaja Gangasingh Dental College Ganagapur (Rajasthan) \\ ${ }^{1}$ Reader, Prosthodontic Maharaja Gangasingh Dental College Ganagapur (Rajasthan)
}

\begin{abstract}
Vertical dimension can be described best as the vertical height of the face. It is determined by muscular relationships, utilizing the physiologic rest position of the mandible as the guiding factor. Therefore, a practical knowledge of the physiologic rest position is essential in the determination of correct vertical dimension of occlusion.Vertical dimension of occlusion is not usually a major concern in routine fixed porosthodontic treatment. Patients with severe tooth wear, however, may require extensive restorative treatment to regain appropriate function, esthetics and comfort. Treatment of a reduced vertical dimension is not designed to increase the vertical dimension beyond normal, but is intended to restore the amount of vertical dimension that has been lost. In rehabilitative procedures, the masticatory organ must be reconstructed within the limits of the physiologic rest position with sufficient allowance for a functional interocclusal distance. Management of a case of severe tooth wear caused by loss of posterior tooth support is described.

Key words: Amelogenesis imperfecta, Dentinogenesis imperfecta, Bruxism, Vertical dimension of occlusion.
\end{abstract}

\section{Introduction}

A patient's vertical dimension has a significant effect on his/her daily function and esthetics. Proper vertical dimension enables uniform force distribution to prevent undue stress to the neuromuscular region. However, vertical dimension of occlusion may be compromised in patients with severe tooth wear which may be due to Dentinogenesis imperfecta/Amelogenesis imperfecta, parafunctional habits, loss of posterior tooth support, abrasion, erosion, and wear from opposing restorative materials $(1,2,3)$. When compromised vertical dimension is often treated with surgical and orthognathic procedures this may cause patient anxiety and/or refusal of treatment. Thus, vertical dimension can in limited occasions, be altered via prosthodontic means $(4,5$, $6)$.

\section{Case Presentation}

A 62 year old male patient visited the prosthodontic department of government dental college and hospital, Patiala with the chief complaint of inability to chew since 3 year and impaired appearance of teeth.

Patient presented with a history of missing teeth \#18,17,16,26,27,28,36,46,47, severe deep bite ,severe attrition on lingual surfaces of maxillary premolars and inciso-labial surfaces of mandibular incisors, moderate attrition on occlusal surfaces of mandibular premolars and canines and lingual surfaces of maxillary anterior teeth, grade III furcation involvement with 37 and gross caries with 37, 38, 47 and 48 .

A comprehensive diagnostic evaluation, records and photographs were utilized to initiate a treatment plan. Full mouth restoration at restored vertical dimension was evaluated as a modality to achieve more ideal aesthetics, function and health (7). The neuromuscular system had to be harmonized with TMJ and it was necessary for making $\mathrm{CR}$ to coincide with $\mathrm{CO}$.

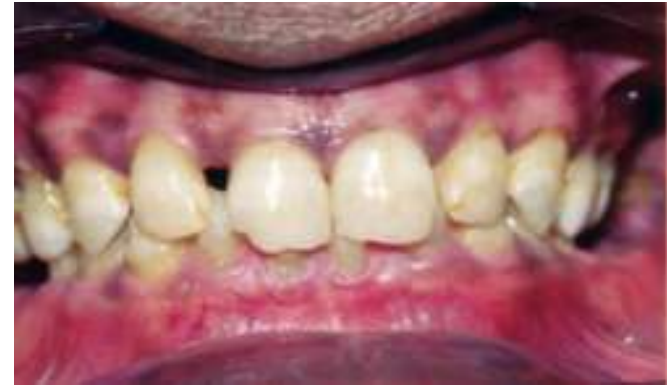

PRE-TREATMENT

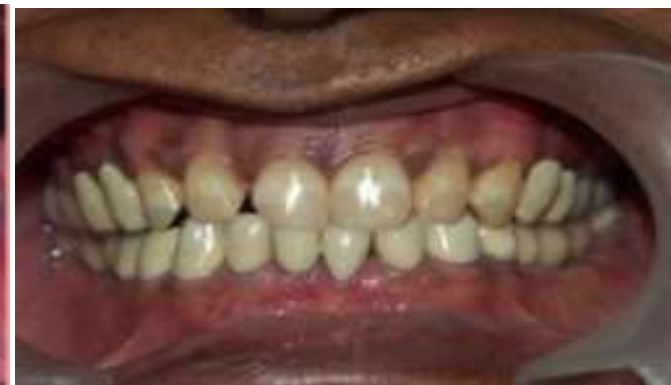

POST-TREATMENT 


\section{Treatment Plan}

Included extraction of tooth 37 ,root canais of $14,15,24,25,31,32,33,37,38,41,42,43,45$ and 48 . Oral prophylaxis, maxillary occlusal plane correction to smile line, treatment RPD's including 17, $16,26,27,36,37,46,47$ with $\mathrm{CO}$ coinciding with $\mathrm{CR}$ at an increased VDO for a period of 2 months. After evaluation of patient 's response to restored VDO-restoration of lingual surfaces of maxillary premolars and mandibular central incisors with composite resins, diagnostic wax-up, provisionals, tooth preparations, provisionals cementation for 1 month, final restorations(metal ceramic crowns with $14,15,24,25,31,32,33,41,42,43$,FPDs with 34 through 38 and 44 through 48,RPD with 16,17,26,27.),group function occlusal scheme and cusp fossa occlusal relation $(8,9)$.

\section{Pre-Treatment Phase}

After required extraction and oral prophylaxis ,patient's response to increase in VDO was evaluated through treatment RPD's for a period of 2 months .Treatment RPD's were constructed for $17,16,26,27,36,37,46,47$ by raising VDO by $2 \mathrm{~mm}$ which the patient wore comfortably for a period of 1 month. Maxillary treatment RPD was remade by further increasing VDO by $2 \mathrm{~mm}$ and was evaluated for 1 month. Patient responded favorably

-Lucia jig was made at this increased VDO to serve as a guide to planned VD and recording of CR.

-Lingual surfaces of maxillary premolars and mandibular incisors were restored with composite resins.

-Diagnostic wax-up was completed on a set of study models, which were mounted on hanau semi-adjustable articulator with CR record made at the estimated VDO .Right lateral, left lateral and protrusive records were made to set articulator controls. (Fig.1)Diagnostic tooth preparations and wax-up was done on the casts keeping in mind establishment of maxillary aesthetic occlusal plane. (Fig 2).

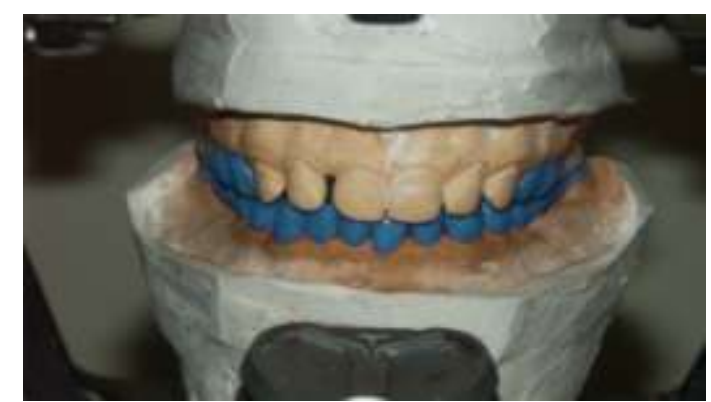

Fig 1

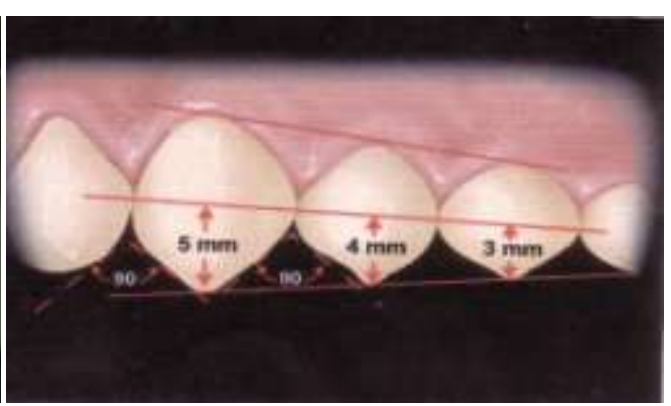

Fig 2

\section{Diagnostic Wax-Up}

Started on mandibular anterior teeth to establish a favorable anterior guidance .Maxillary premolars were waxed up next according to predetermined aesthetic occlusal plane. Wax up of maxillary RPD was done to serve as a guide for wax-up of lower teeth. Wax-up of lower posterior teeth was done last ,one quadrant at a time checking for occlusion in all excursions. Maxillary wax-up was surveyed for designing of RPD.

\section{Provisionalization}

Diagnostic wax-up was used to fabricate provisionalrestorations to verify patient's

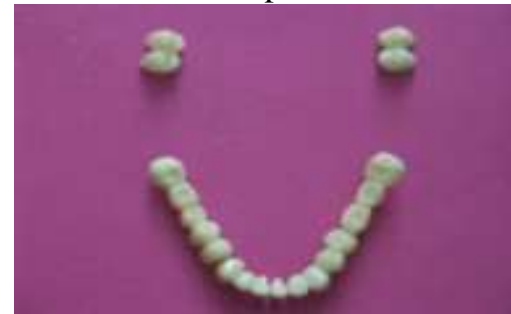

adaptation and response to new vertical guidelines and planned restorations (10).

\section{Final Preparations}

Tooth preparations were done in single sitting in patient's mouth. (Fig 3) Addition silicone, elastomeric impression material, final impressions were made. Provisionals were cemented and equilibrated. (Fig 4)Also, anterior guidance was refined .Patient reviewed after 6 weeks and was found to be comfortable. Occlusal harmony was periodically re-evaluated as muscles relaxed. 


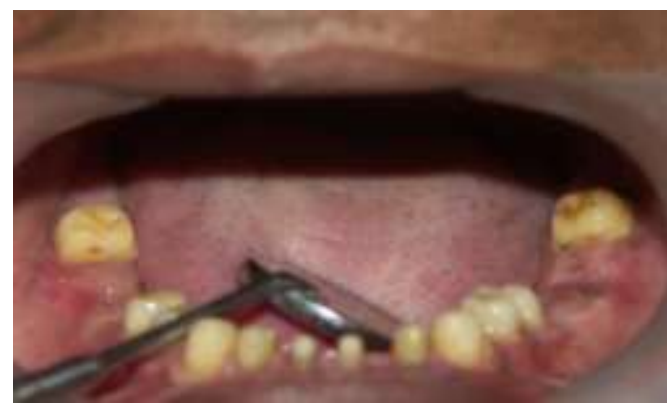

Fig 3

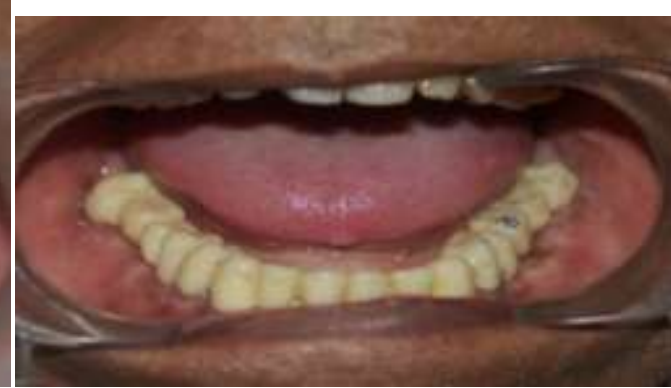

Fig 4

\section{Final Wax-Up}

Master casts were mounted on Hanau articulator using spring bow and CR record. Wax-up for final restorations was done conforming them to diagnostic wax-up as closely as possible.(Fig 5)Maxillary wax-up was surveyed for designing of RPD.

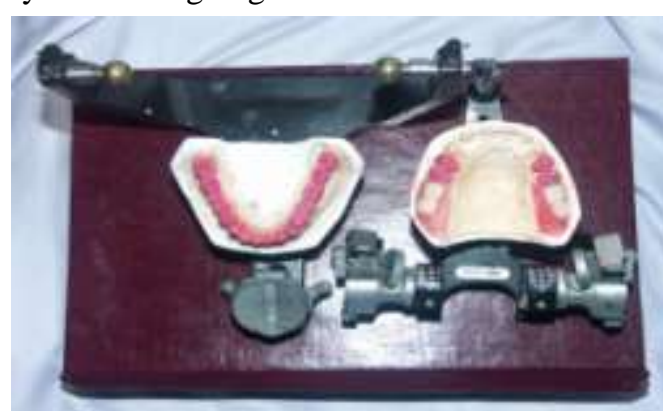

Fig 5

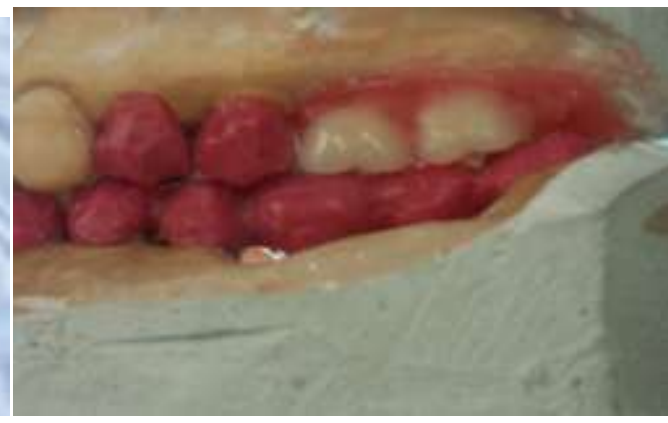

CUSP-FOSSA RELATION

\section{Final Restorations}

Castings were seated and occlusion was refined on the articulator.

Castings adjusted in patient's mouth. (Fig 6)

Final metal ceramic crowns and FPD's cemented with temporary cement for a period of 1 month.

Maxillary RPD inserted and occlusion refined. (Fig 7)

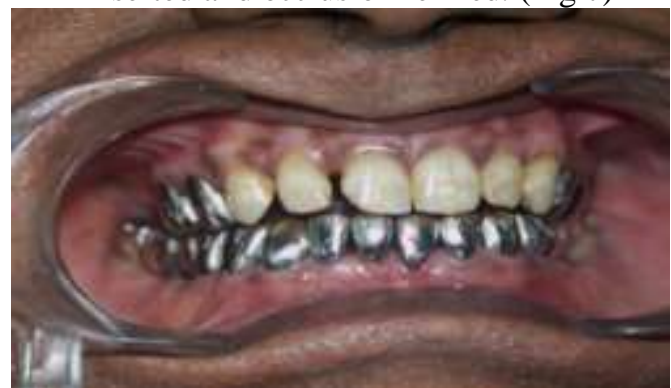

Fig 6

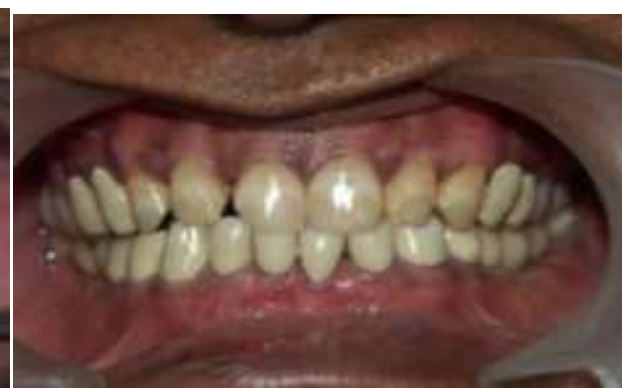

Fig 7

\section{References:}

[1] Dawson PE; Evaluation, diagnosis and treatment of occlusal problems". CV Mosby co, $2^{\text {nd }}$ Edition, 1989.

[2] Okeson JP;" Management of temporo-mandibular disorders and occlusion .Mosby $4^{\text {th }}$ edition.

[3] Chu FCS, yip HK, Newsome PRH, Chow TW, Smales RJ. Restorative management of the worn dentition: 1. Aetiology and diagnosis .Dental Update 2002; 29; 162-68.

[4] Chu FCS, yip HK, Newsome PRH ,Chow TW, Smales RJ .Restorative management of the worn dentition 2.Localised anterior toothwear. Dental update 2002; 29; 214-22.

[5] Chu FCS, yip HK, Newsome PRH, Chow TW, Smales RJ. Restorative management of the worn detition.3. Localized posterior toothwear. Dental update 2002; 29;267-72.

[6] Chu FCS, yip HK, Newsome PRH, Chow TW, Smales RJ .Restorative management of the worn detition.4. Generalized toothwear. Dental Update 2002; 29; 318-24.

[7] Binkley TK, Binkley CJ. A practical approach to full mouth rehabilitation .j Prosthet dent 1987;57;261-65

[8] Hunt PR. A classification and scheme of therapy for occlusal breakdown .Quintessence International 1985; 5; 321-31.

[9] Wassell RW, Steele JG .considerations when planning occlusal rehabilitation: a review of literature .Int dent j 1998; 48:571-81.

[10] Ahuja P, Prakash H, Gupta V. Occlusal rehabilitation: clinical considerations and case report. Journal of Indian Prosthodontic Society.1999; 10:25-30. 